\title{
MAD1L1 and TSNARE gene polymorphisms are associated with schizophrenia susceptibility in the Han Chinese population
}

\author{
Xianglai Liu ${ }^{1 *}{ }^{*}$, Hailing Xie ${ }^{2 \dagger}$, Zejuan Fu ${ }^{3 \dagger}$, Qiankun Yao ${ }^{4 \dagger}$, Tianming Han ${ }^{1 \dagger}$, Dafei Zhan ${ }^{1}$, Zhan Lin ${ }^{1}$ and
} Hong Zhu'

\begin{abstract}
Background: Schizophrenia (SCZ) is a severe mental illness with high heritability. This study aimed to explore the correlation between MAD1L1, TSNARE polymorphisms and SCZ susceptibility.

Methods: A total of 493 SCZ patients and 493 healthy controls were included. The genotypes of MAD1L1 and TSNARE polymorphisms were identified by Agena MassARRAY platform. Odds ratio (OR) and 95\% confidence intervals (Cls) were tested via logistic regression analysis in multiple genetic models and different subgroups.

Results: We observed that AG genotype of rs1107592, AG genotype of rs4976976, and CA genotype of rs67756423 decreased the susceptibility to SCZ ( $p<0.05)$. Age stratification analysis showed that the TC genotype of rs 12666575 , AG genotype of rs 1 107592, and AG genotype of rs4976976 decreased the risk of SCZ individuals older than 36 years $(p<0.05)$. In addition, the AG and AA genotype of rs4976976, the CA genotype of rs67756423 were associated with a lower risk of SCZ in males $(p<0.05)$. In females, the TT genotype of rs 12666575 in recessive model, the AG and AA-AG genotype of rs 1107592 in heterozygote and dominant model, could reduce the susceptibility to SCZ $(p<0.05)$. However, no significant association was found after Bonferroni correction.
\end{abstract}

Conclusions: Our results suggest that MAD1L1 and TSNARE genetic polymorphisms exert a protective role in the risk of SCZ. These findings provide evidence that MADIL1 and TSNARE may serve as potential biomarkers of SCZ. However, a replication experiment in a cohort with large sample size are required to confirm our findings.

Trial registration Not applicable.

Keywords: Schizophrenia, MAD1L1, TSNARE, Susceptibility

\section{Background}

Schizophrenia (SCZ) is a mental illness characterized by hallucinations, delusions, emotional disorders, and social withdrawal [1]. SCZ causes some suffering for individuals and poses a huge psychosocial and economic burden

\footnotetext{
${ }^{*}$ Correspondence: liuxianglailx|@163.com

${ }^{\dagger}$ Xianglai Liu, Hailing Xie, Zejuan Fu, Qiankun Yao and Tianming Han are co-first authors

${ }^{1}$ Institute of Mental Health, Hainan Provincial Anning Hospital, No 10, Nanhai Avenue East, Haikou 571100, Hainan, China

Full list of author information is available at the end of the article
}

to families and societies. Saha et al. reported a median incidence of 15.2 per 100,000 persons and a lifetime prevalence of $0.4-1 \%$ of the general population [2]. In 2010 there were 7.16 million people in China affected by SCZ during their lifetime, an increase of $132 \%$ compared with 1990. And the prevalence of SCZ in urban China was $0.39 \%$ in $1990,0.57 \%$ in 2000 , and $0.83 \%$ in 2010 [3]. However, the pathogenesis of SCZ is not clear. Recently, many studies have indicated that genetic factors were important in the development of SCZ $[4,5]$. And Cardno et al. reported that the heritability of SCZ is up to $80 \%$ 
[6]. Previous genetic studies have identified some candidate genes (AKT1, 5-HTT, COMT) as risk genes for SCZ to illustrate the biological mechanism of this disorder [5]. However, the exact roles of these candidate genes in SCZ pathogenesis were not fully established.

Mitotic arrest deficient-like 1 (MAD1L1) is a component of the mitotic spindle-assembly checkpoint which prevents the onset of anaphase until all the chromosomes are properly aligned at the metaphase plate [7]. MAD1L1 involved in tumor suppression and cell cycle control. A large body of literature has demonstrated that the expression of MAD1L1 is abnormal in breast cancer, smallcell lung cancer, and other cancers $[8,9]$. Besides, it was found that MAD1L1 was related to the reward systems functioning in healthy adults [10]. In a recent study found that MAD1L1 antigene showed increased IgG level in SCZ patients compared with control subjects [11]. Zhao et al. indicated that single nucleotide polymorphism (SNP) in MAD1L1 was significantly associated with bipolar disorder in Chinese people [12]. Nevertheless, there are few studies on the role of MAD1L1 variants in SCZ development.

T-SNARE domain-containing 1 gene (TSNARE1) may have evolved from the harbinger transposon superfamily within the vertebrate lineage [13]. It has been suggested that TSNARE possesses functions related to transcriptional regulation, nuclear import, and DNA binding [14]. Then, bioinformatic predictions indicated it may bind SNARE and have SNAP receptor activity. Additionally, a genome-wide association study (GWAS) meta-analysis has reported that TSNARE1 rs10098073 and rs4129585 were closely related to SCZ and bipolar susceptibility in Caucasians [15]. This was in line with the discovery of $\mathrm{Gu}$ et al., which indicated a significant correlation between rs10098073, rs4129585 in TSNARE and SCZ risk in Southeast Chinese Han and Zhuang people [16]. However, the relationship between other SNPs polymorphisms in TSNARE and SCZ susceptibility has not been explored in the Northwest Chinese Han population.

In the present study, we mainly focused on the role of MAD1L1 and TSNARE1 in the pathogenesis of SCZ. We evaluated the association of MAD1L1 rs10275045, rs12666575, rs1107592 and TSNARE1 rs4976976, rs67756423 with SCZ risk in the Northwest Chinese Han population. These findings will provide insights into the pathogenesis and development of SCZ.

\section{Methods}

\section{Study subjects}

A total of 986 individuals, which included 493 SCZ patients and 493 controls, were enrolled from Xi'an Mental Health Center. Schizophrenia was identified by two psychiatrists on the basis of the Tenth Revision of
Table 1 Characteristics of cases and controls

\begin{tabular}{llll}
\hline Variables & Cases $(\mathbf{n}=\mathbf{4 9 3})$ & Controls $(\mathbf{n}=\mathbf{4 9 3})$ & $\boldsymbol{p}$ \\
\hline Age, year (mean \pm SD) & $36.47 \pm 13.20$ & $36.50 \pm 11.89$ & $0.968^{\mathrm{a}}$ \\
$>36$ & $220(44.6 \%)$ & $230(46.7 \%)$ & \\
$\leq 36$ & $273(55.4 \%)$ & $263(53.3 \%)$ & \\
Gender & & & $0.799^{\mathrm{b}}$ \\
Male & $261(52.9 \%)$ & $257(52.1 \%)$ & \\
Female & $236(47.1 \%)$ & $232(47.9 \%)$ & \\
\hline
\end{tabular}

SD: standard deviation

$p^{\mathrm{a}}$ values were calculated from student's $t$ test

$p^{\mathrm{b}}$ values were calculated from $\mathrm{X}^{2}$ test

$p<0.05$ indicates statistical difference

International Classification of Diseases. Patients who met the following conditions were excluded: (1) mental diseases induced by organic brain syndrome, (2) neurological diseases, (3) mental retardation, (4) severe brain injury, (5) non-cooperating patients with superexcitation, (6) pregnant or breastfeeding women. Inclusion criteria for healthy controls were individuals without family history of mental disorder, severe head injury, febrile convulsion in childhood or infant stage. Moreover, we used G*power software to calculate the minimal required sample size based on the probability of a typeIerror of alpha $=5 \%$, typeIIerror of beta $=15 \%$ (power $=85 \%$ ), effect size of 0.2 . This calculation yielded a sample consisting of at least 450 cases and 450 controls. Then, we recruited 493 cases and 493 controls in this study.

The legal guardian of these participants provided informed consent documents on their behalf. This study got approval of the Ethics Committee of Xi'an Mental Health Center and followed the Declaration of Helsinki.

\section{SNP genotyping}

Peripheral blood samples were collected from each subject. DNA was isolated from venous blood sample by the GoldMag DNA purification kit (GoldMag Co. Ltd, $\mathrm{Xi}^{\prime}$ an, China) in accordance with the user's protocol, then quantified by NanoDrop 2000 (Thermo Scientific, Waltham, MA, USA). The SNPs in the MAD1L1, TSNARE1 genes were chosen based on the minor allele frequency (MAF) $>0.05$ in Han Chinese from the 1000 Genome Projects. Three SNPs (rs10275045, rs12666575, rs1107592) in MAD1L1 and two SNPs (rs4976976, rs67756423) in TSNARE1 were selected in the present study.

Primers of the five SNPs are listed in Additional file 1: Table S1. PCR reactions were performed in a buffer containing $1 \mu \mathrm{l}$ DNA, $0.5 \mu \mathrm{l}$ PCR Buffer, $0.4 \mu \mathrm{l} \mathrm{MgCl}, 0.1 \mu \mathrm{l}$ dNTP Mix, $1.0 \mu \mathrm{l}$ primer mix, and $0.2 \mu \mathrm{l}$ Taq ligase in a final reaction volume of $5 \mu$ l. The reaction mixture was 


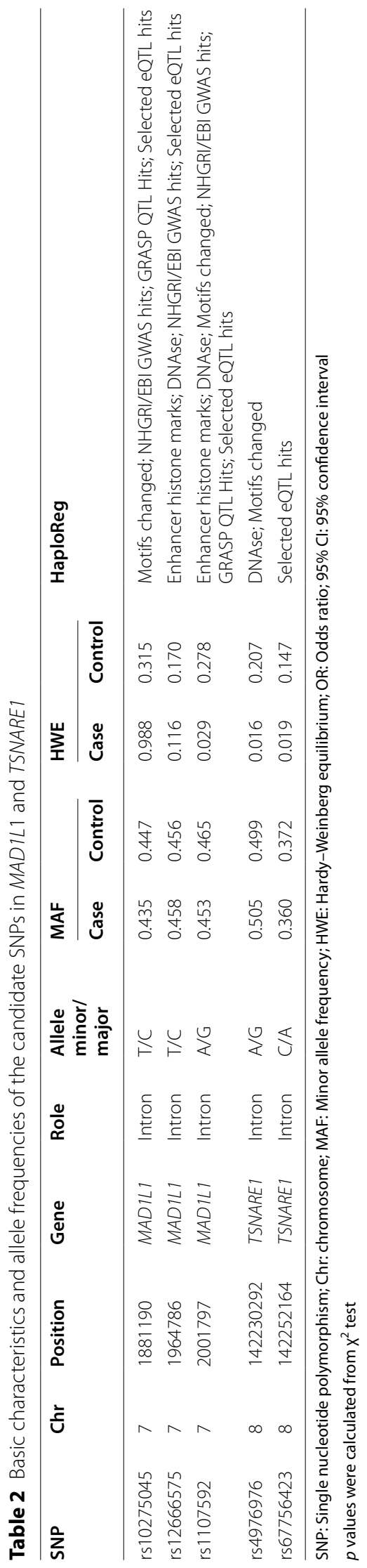


heated to $94{ }^{\circ} \mathrm{C}$ for $15 \mathrm{~min}$ for denaturation. Then, the sample was subjected to 45 cycles of $94{ }^{\circ} \mathrm{C} 20 \mathrm{~s}$, annealing at $56{ }^{\circ} \mathrm{C} 30 \mathrm{~s}$ and extension at $72{ }^{\circ} \mathrm{C} 60 \mathrm{~s}$, followed by a final extension step at $72{ }^{\circ} \mathrm{C}$ for $3 \mathrm{~min}$. The PCR product was used to genotype using the Agena MassArray platform (Agena Bioscience, San Diego, CA, USA). The raw data was analyzed and managed using Agena Typer 4.0 software (Agena Bioscience, San Diego, CA, USA).

\section{Data analysis}

We performed Pearson's $X^{2}$ test and student's t-test to assess the differences in gender and age of study populations, respectively. Hardy-Weinberg equilibrium (HWE) was examined by Pearson's $X^{2}$ test. The distribution of SNP allele and genotype between SCZ patients and healthy controls were tested by $\chi^{2}$ test. Odds ratio (OR) and $95 \%$ confidence intervals (CI) were applied to estimate the relationship between MAD1L1, TSNARE1 gene and SCZ risk by logistic regression analysis in multiple inheritance models and subgroup. We also evaluated the SNP-SNP interaction in the risk of SCZ using multifactor dimensionality reduction (MDR). Statistical power and false positive report probability (FPRP) values were calculated by the Excel spreadsheet which was offered on Wacholder's website [17]. The functional role of these SNPs was predicted by HaploReg database (https:// pubs.broadinstitute.org/mammals/haploreg/haploreg. php). The differences were deemed significant at $p<0.05$, whereas a value of corrected $p<0.05 / 5$ was considered significant after Bonferroni correction.

\section{Results}

\section{Study subjects}

Totally, 493 patients (261 men and 236 women) of SCZ and 493 healthy controls (257 men and 232 women) were enrolled with a mean age of $36.47 \pm 13.20$ years and $36.50 \pm 11.89$ years, respectively (Table 1 ). There were no statistical differences in age $(p=0.968)$ and gender $(p=0.799)$ between the two groups.

\section{Basic information for the candidate SNPs}

Three SNPs (rs10275045, rs12666575, rs1107592) in MAD1L1 and two SNPs (rs4976976, rs67756423) in TSNARE1 were successfully genotyped. In Table 2, we described the details of the selected SNPs regarding SNP ID, gene, chromosomal position, role, MAF. All SNPs were following HWE $(p>0.05)$ and were found in the intron region.

\section{SCZ susceptibility evaluation}

Multiple inheritance models (allele, codominant, dominant, recessive, and additive models) were performed to assess the relationship between SNPs and SCZ susceptibility (Table 3 ). We found that individuals carrying the heterozygous genotype AG in rs1107592 $(\mathrm{OR}=0.72, \quad 95 \% \quad \mathrm{CI}=0.54-0.97, \quad p=0.031), \quad \mathrm{AG}$ in rs4976976 $(\mathrm{OR}=0.73,95 \% \mathrm{CI}=0.54-0.99, p=0.043)$, $\mathrm{CA}$ in rs67756423 $(\mathrm{OR}=0.72,95 \% \mathrm{CI}=0.55-0.94$, $p=0.017$ ) were reduced the susceptibility to SCZ when compared with the GG, GG, and AA genotype. However, no significant association was found after Bonferroni correction. Moreover, the significant association of rs10275045 and rs12666575 in MAD1L1 with SCZ susceptibility was not detected.

Subsequently, we carried out stratification analysis based on age and gender (Table 4). Among people older than 36 years, the TC heterozygote in rs12666575 $(\mathrm{OR}=0.64,95 \% \mathrm{CI}=0.41-1.00, p=0.048)$, the AG heterozygote in $\mathrm{rs} 1107592(\mathrm{OR}=0.62,95 \% \mathrm{CI}=0.40-0.96$, $p=0.034)$, and the AG heterozygote in rs4976976 $(\mathrm{OR}=0.63,95 \% \mathrm{CI}=0.40-0.99, p=0.045)$ had a riskdecreasing effects compared with the CC, GG and GG homozygote. However, no significant association was found after Bonferroni correction.

After stratifying by gender (Table 4), the CA genotype of rs67756423 was associated with a lower risk of SCZ in males $(\mathrm{OR}=0.64,95 \% \mathrm{CI}=0.44-0.92, p=0.017)$. As for rs4976976, the AG and AA genotype were related to a lower incidence of SCZ under codominant (AG vs. AA, $\mathrm{OR}=0.61,95 \% \mathrm{CI}=0.40-0.93, p=0.022)$ and recessive model (AA vs. AG-GG, OR $=1.53,95 \% \mathrm{CI}=1.02-2.29$, $p=0.041$ ). In females, the TT genotype of rs12666575 obviously reduced the susceptibility to $\mathrm{SCZ}$ in recessive model (TT vs. TC-CC, $\mathrm{OR}=0.61,95 \% \mathrm{CI}=0.52-1.15, p=0.042$ ). Rs1107592 decreased the risk of SCZ in codominant (AG vs. $\mathrm{GG}, \mathrm{OR}=0.62,95 \% \mathrm{CI}=0.40-0.95, p=0.029$ ) and dominant models (AA-AG vs. GG, OR $=0.65,95 \%$ $\mathrm{CI}=0.43-0.98, p=0.038$ ). However, no significant association was found after Bonferroni correction.

\section{MDR analysis}

Finally, we examined the interaction of SNP-SNP using MDR. The Dendrogram and Fruchterman-Reingold of SNP-SNP interactions were exhibited in Fig. 1a and b. As is shown in Table 5, rs67756423 was the single model to forecast SCZ risk (testing accuracy $=0.509, \mathrm{CVC}=6 / 10$, $p=0.004)$. The two-locus model included rs10275045 and rs1107592 (testing accuracy $=0.508, C V C=4 / 10$, $p<0.0001)$. The three-locus model was the combination of rs10275045, rs1107592, and rs4976976 (testing accuracy $=0.550, C V C=10 / 10, p<0.0001)$. The four-locus model comprised of rs10275045, rs1107592, rs4976976, and rs67756423 (testing accuracy $=0.548, C V C=10 / 10$, $p<0.0001) . \quad \mathrm{Rs} 10275045, \quad \mathrm{rs} 12666575, \quad \mathrm{rs} 1107592$, rs4976976, rs67756423 were constituted five-locus model (testing accuracy $=0.522, \quad \mathrm{CVC}=10 / 10, \quad p<0.0001$ ). 
Table 3 Relationship of polymorphisms in MAD1L1 and TSNARE1 genes and SCZ susceptibility

\begin{tabular}{|c|c|c|c|c|c|}
\hline Gene & SNP & Model & Genotype & OR $(95 \% \mathrm{Cl})$ & $p$ \\
\hline \multirow[t]{10}{*}{ MAD1L1 } & rs10275045 & Allele & C & 1.00 & \\
\hline & & & $\mathrm{T}$ & $\begin{array}{l}0.95 \\
(0.80-1.14)\end{array}$ & 0.589 \\
\hline & & Codominant & $\mathrm{CC}$ & 1.00 & \\
\hline & & & $\mathrm{TT}$ & $\begin{array}{l}0.93 \\
(0.64-1.34)\end{array}$ & 0.680 \\
\hline & & & $\mathrm{TC}$ & $\begin{array}{l}0.87 \\
(0.65-1.16)\end{array}$ & 0.334 \\
\hline & & Dominant & $\mathrm{CC}$ & 1.00 & \\
\hline & & & TT-TC & $\begin{array}{l}0.88 \\
(0.67-1.16)\end{array}$ & 0.372 \\
\hline & & Recessive & $\mathrm{TC}-\mathrm{CC}$ & 1.00 & \\
\hline & & & $\mathrm{TT}$ & $\begin{array}{l}1.01 \\
(0.73-1.39)\end{array}$ & 0.946 \\
\hline & & Additive & - & $\begin{array}{l}0.95 \\
(0.79-1.14)\end{array}$ & 0.580 \\
\hline \multirow[t]{10}{*}{ MAD1L1 } & rs12666575 & Allele & C & 1.00 & \\
\hline & & & $\mathrm{T}$ & $\begin{array}{l}1.01 \\
(0.85-1.21)\end{array}$ & 0.909 \\
\hline & & Codominant & $\mathrm{CC}$ & 1.00 & \\
\hline & & & $\mathrm{TT}$ & $\begin{array}{l}1.07 \\
(0.75-1.53)\end{array}$ & 0.721 \\
\hline & & & $\mathrm{TC}$ & $\begin{array}{l}0.78 \\
(0.58-1.05)\end{array}$ & 0.101 \\
\hline & & Dominant & CC & 1.00 & \\
\hline & & & TT-TC & $\begin{array}{l}0.86 \\
(0.65-1.13)\end{array}$ & 0.279 \\
\hline & & Recessive & $\mathrm{TC}-\mathrm{CC}$ & 1.00 & \\
\hline & & & $\mathrm{TT}$ & $\begin{array}{l}1.24 \\
(0.91-1.70)\end{array}$ & 0.166 \\
\hline & & Additive & - & $\begin{array}{l}1.01 \\
(0.84-1.21)\end{array}$ & 0.920 \\
\hline \multirow[t]{10}{*}{ MAD1L1 } & rs1107592 & Allele & G & 1.00 & \\
\hline & & & A & $\begin{array}{l}0.96 \\
(0.80-1.14)\end{array}$ & 0.616 \\
\hline & & Codominant & GG & 1.00 & \\
\hline & & & AA & $\begin{array}{l}0.96 \\
(0.67-1.37)\end{array}$ & 0.820 \\
\hline & & & $A G$ & $\begin{array}{l}0.72 \\
(0.54-0.97)\end{array}$ & 0.031 \\
\hline & & Dominant & GG & 1.00 & \\
\hline & & & $A A-A G$ & $\begin{array}{l}0.79 \\
(0.60-1.04)\end{array}$ & 0.093 \\
\hline & & Recessive & $A G-G G$ & 1.00 & \\
\hline & & & $\mathrm{AA}$ & $\begin{array}{l}1.17(0.86- \\
1.59)\end{array}$ & 0.308 \\
\hline & & Additive & - & $\begin{array}{l}0.96 \\
(0.80-1.14)\end{array}$ & 0.625 \\
\hline
\end{tabular}

Table 3 (continued)

\begin{tabular}{|c|c|c|c|c|c|}
\hline Gene & SNP & Model & Genotype & OR $(95 \% \mathrm{Cl})$ & $p$ \\
\hline \multirow[t]{10}{*}{ TSNARE1 } & rs4976976 & Allele & G & 1.00 & \\
\hline & & & A & $\begin{array}{l}1.03 \\
(0.86-1.22)\end{array}$ & 0.787 \\
\hline & & Codominant & GG & 1.00 & \\
\hline & & & $\mathrm{AA}$ & $\begin{array}{l}1.05 \\
(0.74-1.49)\end{array}$ & 0.801 \\
\hline & & & $A G$ & $\begin{array}{l}0.73 \\
(0.54-0.99)\end{array}$ & 0.043 \\
\hline & & Dominant & GG & 1.00 & \\
\hline & & & $A A-A G$ & $\begin{array}{l}0.83 \\
(0.62-1.10)\end{array}$ & 0.192 \\
\hline & & Recessive & AG-GG & 1.00 & \\
\hline & & & $\mathrm{AA}$ & $\begin{array}{l}1.29 \\
(0.97-1.72)\end{array}$ & 0.083 \\
\hline & & Additive & - & $\begin{array}{l}1.02 \\
(0.86-1.22)\end{array}$ & 0.790 \\
\hline \multirow[t]{10}{*}{ TSNAREI } & rs67756423 & Allele & A & 1.00 & \\
\hline & & & G & $\begin{array}{l}0.95 \\
(0.79-1.14)\end{array}$ & 0.576 \\
\hline & & Codominant & $\mathrm{AA}$ & 1.00 & \\
\hline & & & CC & $\begin{array}{l}1.10 \\
(0.74-1.62)\end{array}$ & 0.641 \\
\hline & & & $C A$ & $\begin{array}{l}0.72 \\
(0.55-0.94)\end{array}$ & 0.017 \\
\hline & & Dominant & $\mathrm{AA}$ & 1.00 & \\
\hline & & & CC-CA & $\begin{array}{l}0.79 \\
(0.62-1.03)\end{array}$ & 0.077 \\
\hline & & Recessive & CA-AA & 1.00 & \\
\hline & & & $\mathrm{CC}$ & $\begin{array}{l}1.31 \\
(0.91-1.88)\end{array}$ & 0.151 \\
\hline & & Additive & - & $\begin{array}{l}0.95 \\
(0.79-1.14)\end{array}$ & 0.587 \\
\hline
\end{tabular}

SNP: single nucleotide polymorphism; OR, odds ratio; $95 \% \mathrm{Cl}$, $95 \%$ confidence interval

$p$ values were calculated by logistic regression analysis with adjustments for age and gender

Bold values indicate statistical significance $(p<0.05)$

Therefore, the best model was the three-locus model, a combination of rs10275045, rs1107592, and rs4976976, with the highest testing accuracy and perfect CVC.

\section{FPRP analysis}

FPRP and statistical power were calculated for all positive results. As shown in Table 6, at the prior probability of 0.25 and FPRP threshold of 0.2 , all significant results of MAD1L1 and TRNARE polymorphisms remained noteworthy. 
Table 4 Relationships of MAD1L1 and TSNARE1 polymorphisms with SCZ risk stratified by age and gender

\begin{tabular}{|c|c|c|c|c|c|c|c|c|c|c|}
\hline \multirow[t]{2}{*}{ Gene SIP } & \multirow[t]{2}{*}{ Model } & \multirow[t]{2}{*}{ Genotype } & \multicolumn{2}{|l|}{$\leq 36$} & \multicolumn{2}{|l|}{$>36$} & \multicolumn{2}{|l|}{ Male } & \multicolumn{2}{|l|}{ Female } \\
\hline & & & OR $(95 \% \mathrm{Cl})$ & $p$ & OR $(95 \% \mathrm{Cl})$ & $p$ & OR $(95 \% \mathrm{Cl})$ & $p$ & OR $(95 \% \mathrm{Cl})$ & $p$ \\
\hline \multirow{10}{*}{$\begin{array}{l}\text { MAD1L1 } \\
\text { rs10275045 }\end{array}$} & \multirow[t]{2}{*}{ Allele } & C & 1.00 & & 1.00 & & 1.00 & & 1.00 & \\
\hline & & $\mathrm{T}$ & $0.91(0.71-1.16)$ & 0.429 & $1.01(0.78-1.32)$ & 0.937 & $0.85(0.67-1.09)$ & 0.201 & $1.08(0.83-1.40)$ & 0.575 \\
\hline & \multirow[t]{3}{*}{ Codominant } & $\mathrm{CC}$ & 1.00 & & 1.00 & & 1.00 & & 1.00 & \\
\hline & & $\pi T$ & $0.81(0.50-1.33)$ & 0.404 & $1.05(0.60-1.83)$ & 0.878 & $0.74(0.44-1.24)$ & 0.250 & $1.16(0.69-1.97)$ & 0.572 \\
\hline & & TC & $1.03(0.70-1.52)$ & 0.883 & $0.66(0.43-1.03)$ & 0.065 & $0.70(0.47-1.05)$ & 0.082 & $1.08(0.71-1.63)$ & 0.730 \\
\hline & \multirow[t]{2}{*}{ Dominant } & $\mathrm{CC}$ & 1.00 & & 1.00 & & 1.00 & & 1.00 & \\
\hline & & TT-TC & $0.96(0.67-1.39)$ & 0.838 & $0.75(0.49-1.13)$ & 0.171 & $0.71(0.48-1.04)$ & 0.079 & $1.10(0.74-1.63)$ & 0.634 \\
\hline & \multirow[t]{2}{*}{ Recessive } & TC-CC & 1.00 & & 1.00 & & 1.00 & & 1.00 & \\
\hline & & $\mathrm{TT}$ & $0.80(0.52-1.23)$ & 0.304 & $1.36(0.84-2.21)$ & 0.207 & $0.93(0.60-1.45)$ & 0.747 & $1.11(0.70-1.77)$ & 0.649 \\
\hline & Additive & - & $0.92(0.72-1.17)$ & 0.477 & $0.97(0.74-1.28)$ & 0.855 & $0.84(0.65-1.08)$ & 0.179 & $1.08(0.83-1.40)$ & 0.568 \\
\hline \multirow{10}{*}{$\begin{array}{l}\text { MAD1L1 } \\
\text { rs12666575 }\end{array}$} & \multirow[t]{2}{*}{ Allele } & C & 1.00 & & 1.00 & & 1.00 & & 1.00 & \\
\hline & & $\mathrm{T}$ & $0.98(0.77-1.24)$ & 0.853 & $1.05(0.81-1.37)$ & 0.707 & $0.95(0.74-1.22)$ & 0.691 & $1.08(0.83-1.40)$ & 0.566 \\
\hline & \multirow[t]{3}{*}{ Codominant } & CC & 1.00 & & 1.00 & & 1.00 & & 1.00 & \\
\hline & & TT & $0.99(0.61-1.61)$ & 0.974 & $1.17(0.68-2.02)$ & 0.563 & $0.92(0.56-1.50)$ & 0.728 & $1.29(0.76-2.18)$ & 0.342 \\
\hline & & $\mathrm{TC}$ & $0.91(0.61-1.35)$ & 0.645 & $0.64(0.41-1.00)$ & 0.048 & $0.86(0.57-1.30)$ & 0.472 & $0.70(0.46-1.06)$ & 0.093 \\
\hline & \multirow[t]{2}{*}{ Dominant } & $\mathrm{CC}$ & 1.00 & & 1.00 & & 1.00 & & 1.00 & \\
\hline & & TT-TC & $0.93(0.64-1.36)$ & 0.722 & $0.77(0.51-1.16)$ & 0.210 & $0.88(0.60-1.29)$ & 0.504 & $0.83(0.56-1.24)$ & 0.371 \\
\hline & \multirow[t]{2}{*}{ Recessive } & TC-CC & 1.00 & & 1.00 & & 1.00 & & 1.00 & \\
\hline & & $\mathrm{TT}$ & $1.05(0.69-1.60)$ & 0.817 & $1.55(0.97-2.48)$ & 0.064 & $1.01(0.66-1.54)$ & 0.975 & $0.61(0.52-1.15)$ & 0.042 \\
\hline & Additive & - & $0.99(0.78-1.26)$ & 0.923 & $1.04(0.79-1.36)$ & 0.791 & $0.95(0.74-1.22)$ & 0.682 & $1.08(0.83-1.40)$ & 0.560 \\
\hline \multirow{10}{*}{$\begin{array}{l}\text { MAD1L1 } \\
\text { rs1107592 }\end{array}$} & Allele & G & 1.00 & 0.669 & 1.00 & & 1.00 & & 1.00 & \\
\hline & & A & $0.95(0.75-1.21)$ & & $0.97(0.75-1.26)$ & 0.809 & $1.08(0.84-1.37)$ & 0.561 & $0.84(0.65-1.09)$ & 0.185 \\
\hline & Codominant & GG & 1.00 & & 1.00 & & 1.00 & & 1.00 & \\
\hline & & $\mathrm{AA}$ & $0.92(0.57-1.49)$ & 0.731 & $0.99(0.59-1.69)$ & 0.980 & $1.20(0.74-1.94)$ & 0.452 & $0.73(0.43-1.23)$ & 0.239 \\
\hline & & $A G$ & $0.82(0.55-1.21)$ & 0.312 & $0.62(0.40-0.96)$ & 0.034 & $0.82(0.55-1.22)$ & 0.325 & $0.62(0.40-0.95)$ & 0.029 \\
\hline & Dominant & GG & 1.00 & & 1.00 & & 1.00 & & 1.00 & \\
\hline & & AA-AG & $0.85(0.59-1.22)$ & 0.376 & $0.72(0.47-1.09)$ & 0.120 & $0.93(0.64-1.34)$ & 0.691 & $0.65(0.43-0.98)$ & 0.038 \\
\hline & Recessive & AG-GG & 1.00 & & 1.00 & & 1.00 & & 1.00 & \\
\hline & & $\mathrm{AA}$ & $1.04(0.68-1.58)$ & 0.860 & $1.35(0.86-2.11)$ & 0.193 & $1.35(0.89-2.06)$ & 0.157 & $1.00(0.6-1.56)$ & 0.992 \\
\hline & Additive & - & $0.94(0.74-1.20)$ & 0.635 & $0.97(0.75-1.26)$ & 0.827 & $1.07(0.84-1.36)$ & 0.580 & $0.84(0.64-1.09)$ & 0.179 \\
\hline TSNARE1 & Allele & G & 1.00 & & 1.00 & & 1.00 & & 1.00 & \\
\hline rs4976976 & & $A$ & $0.98(0.77-1.24)$ & 0.859 & $0.92(0.71-1.20)$ & 0.540 & $1.04(0.81-1.33)$ & 0.754 & $1.10(0.85-1.42)$ & 0.472 \\
\hline & Codominant & GG & 1.00 & & 1.00 & & 1.00 & & 1.00 & \\
\hline & & $\mathrm{AA}$ & $0.97(0.60-1.55)$ & 0.884 & $0.85(0.50-1.44)$ & 0.551 & $1.10(0.67-1.80)$ & 0.715 & $1.19(0.72-1.97)$ & 0.490 \\
\hline & & $A G$ & $0.73(0.48-1.10)$ & 0.136 & $0.63(0.40-0.99)$ & 0.045 & $0.61(0.40-0.93)$ & 0.022 & $0.96(0.62-1.50)$ & 0.865 \\
\hline & Dominant & GG & 1.00 & & 1.00 & & 1.00 & & 1.00 & \\
\hline & & $A A-A G$ & $0.80(0.54-1.18)$ & 0.268 & $0.70(0.46-1.07)$ & 0.095 & $0.74(0.49-1.10)$ & 0.136 & $1.04(0.69-1.57)$ & 0.852 \\
\hline & Recessive & AG-GG & 1.00 & & 1.00 & & 1.00 & & 1.00 & \\
\hline & & $\mathrm{AA}$ & $1.19(0.80-1.75)$ & 0.397 & $1.15(0.74-1.78)$ & 0.537 & $0.53(0.42-0.89)$ & 0.041 & $1.22(0.81-1.84)$ & 0.340 \\
\hline & Additive & - & $0.98(0.77-1.24)$ & 0.868 & $0.92(0.70-1.19)$ & 0.507 & $1.04(0.82-1.33)$ & 0.742 & $1.09(0.85-1.40)$ & 0.488 \\
\hline TSNARE 1 & Allele & A & 1.00 & & 1.00 & & 1.00 & & 1.00 & \\
\hline rs67756423 & & $C$ & $0.97(0.75-1.24)$ & 0.787 & $0.93(0.71-1.22)$ & 0.600 & $0.90(0.69-1.16)$ & 0.399 & $1.01(0.78-1.32)$ & 0.931 \\
\hline & Codominant & $\mathrm{AA}$ & 1.00 & & 1.00 & & 1.00 & & 1.00 & \\
\hline & & $\mathrm{CC}$ & $1.13(0.67-1.91)$ & 0.649 & $1.04(0.57-1.89)$ & 0.896 & $1.05(0.60-1.85)$ & 0.854 & $1.15(0.67-2.00)$ & 0.609 \\
\hline & & CA & $0.71(0.49-1.02)$ & 0.065 & $0.71(0.48-1.07)$ & 0.106 & $0.64(0.44-0.92)$ & 0.017 & $0.82(0.55-1.22)$ & 0.321 \\
\hline & Dominant & $\mathrm{AA}$ & 1.00 & & 1.00 & & 1.00 & & 1.00 & \\
\hline & & CC-CA & $0.79(0.56-1.12)$ & 0.190 & $0.78(0.53-1.14)$ & 0.199 & $0.71(0.50-1.01)$ & 0.056 & $0.89(0.61-1.30)$ & 0.548 \\
\hline & Recessive & CA-AA & 1.00 & & 1.00 & & 1.00 & & 1.00 & \\
\hline & & $\mathrm{CC}$ & $1.35(0.83-2.20)$ & 0.229 & $1.25(0.71-2.17)$ & 0.441 & $1.33(0.79-2.26)$ & 0.282 & $1.29(0.77-2.14)$ & 0.333 \\
\hline & Additive & - & $0.96(0.75-1.23)$ & 0.749 & $0.93(0.70-1.22)$ & 0.587 & $0.89(0.69-1.15)$ & 0.390 & $1.01(0.78-1.31)$ & 0.937 \\
\hline
\end{tabular}


Table 4 (continued)

SNP, single nucleotide polymorphism; OR, odds ratio; $95 \% \mathrm{Cl}, 95 \%$ confidence interval

$p$ values were calculated by logistic regression analysis with adjustment for age and gender

Bold values indicate statistical significance $(p<0.05)$

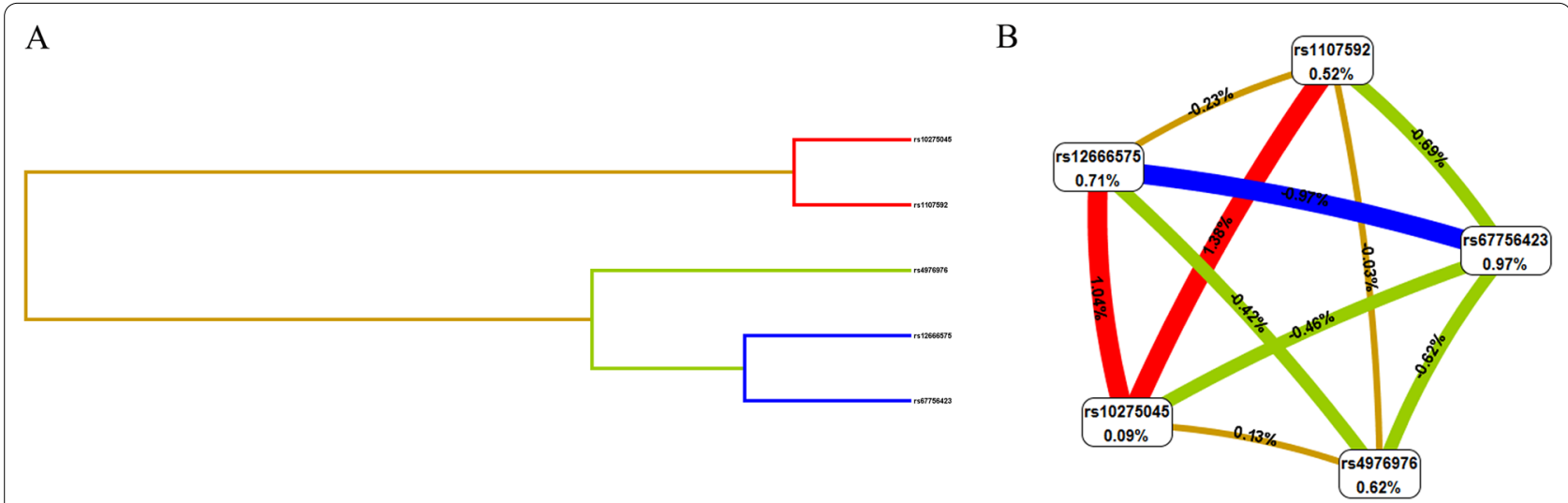

Fig. 1 The Dendrogram and Fruchterman-Reingold of SNP-SNP interaction. a Dendrogram of SNP-SNP interaction. The shorter the line connecting the 2 SNPs, the stronger the interaction. $\mathbf{b}$ Fruchterman-Reingold of SNP-SNP interaction. Each SNP is reported in per cent the value of Information Gain $(I G)$, while numbers in the connections indicate the entropy-based IG for the SNP pairs. Red bar indicates high-level synergies on the phenotype, while the brown indicates a medium-level interaction, green and blue connections with negative IG values indicate redundancy or lack of synergistic interactions between the markers

Table 5 SNP-SNP interaction models of the MAD1L1 and TSNARE1 genes analyzed by the MDR method

\begin{tabular}{|c|c|c|c|c|c|}
\hline Model & Training Bal. Acc & Testing Bal. Acc & CVC & OR $(95 \% \mathrm{Cl})$ & $p$ \\
\hline TSNARE1 rs67756423 & 0.547 & 0.509 & $6 / 10$ & $1.45(1.12-1.86)$ & 0.004 \\
\hline MAD1L1 rs10275045, MAD1L1 rs1107592 & 0.566 & 0.508 & $4 / 10$ & $2.57(1.78-3.71)$ & $<0.0001$ \\
\hline MAD1L1 rs10275045, MAD1L1 rs1107592, TSNARE1 rs4976976 & 0.588 & 0.550 & $10 / 10$ & $2.01(1.56-2.59)$ & $<0.0001$ \\
\hline $\begin{array}{l}\text { MAD1L1 rs10275045, MAD1L1 rs1107592, TSNARE1 rs4976976, TSNAR- } \\
\text { E1rs67756423 }\end{array}$ & 0.607 & 0.548 & $10 / 10$ & $2.41(1.86-3.13)$ & $<0.0001$ \\
\hline $\begin{array}{l}\text { MAD1L1 rs 10275045, MAD1L1 rs12666575, MAD1L1 rs1107592, TSNARE1 } \\
\text { rs4976976, TSNARE1 rs67756423 }\end{array}$ & 0.622 & 0.522 & $10 / 10$ & $2.70(2.09-3.51)$ & $<0.0001$ \\
\hline
\end{tabular}

MDR, multifactor dimensionality reduction; Bal. Acc., balanced accuracy; CVC, cross-validation consistency; OR, odds ratio; $\mathrm{Cl}$, confidence interval $p$ values were calculated using $x^{2}$ tests

$p<0.05$ indicates statistical significance

\section{Discussion}

In this case-control study, our results found that rs1107592 in MAD1L1, rs4976976, and rs67756423 in TSNARE were related to a decreased risk of SCZ in the overall analysis. In addition, MAD1L1-rs12666575, -rs1107592, and TSNARE-rs4976976 significantly decreased the occurrence of SCZ individuals aged $>36$ years. Subsequently, the stratification results based on age were shown that TSNARE-rs4976976, -rs67756423 in males and MAD1L1-rs1107592,rs12666575 in females are associated with a lower risk of SCZ. These results suggested that MAD1L1 and TSNARE genetic polymorphisms were associated with SCZ susceptibility and played a protective role in the development of SCZ.

MAD1L1 is located at human chromosome $7 q 22.3$ and involves cell cycle control and tumor suppression. Recently, some research has reported that MAD1L1 rs12666575 was related to SCZ risk in different genetic backgrounds. For example, Sleiman et al. demonstrated that rs12666575 was associated with SCZ susceptibility in a mixed-ancestry cohorts from Caucasians, African Americans, and Asians in 2013 [15]. A genomewide association study also discovered that rs12666575 reduced the incidence of SCZ in the Swedish sample [18]. 
Table 6 False positive report probability of the association MAD1L1 and TRNARE polymorphisms and SCZ susceptibility

\begin{tabular}{|c|c|c|c|c|c|c|c|c|c|}
\hline \multirow[t]{2}{*}{ Model and variables } & \multirow[t]{2}{*}{ Genotype } & \multirow[t]{2}{*}{ OR $(95 \% \mathrm{Cl})$} & \multirow[t]{2}{*}{$p^{\mathrm{a}}$} & \multirow[t]{2}{*}{ Statistical power } & \multicolumn{5}{|c|}{ Prior probability } \\
\hline & & & & & 0.25 & 0.1 & 0.01 & 0.001 & 0.0001 \\
\hline \multicolumn{10}{|l|}{ Overall analysis } \\
\hline rs1107592 A>G & $A G$ vs GG & $0.72(0.54-0.97)$ & 0.031 & 0.992 & $0.085^{b}$ & 0.218 & 0.754 & 0.969 & 0.997 \\
\hline rs4976976 A>G & $A G$ vs $G G$ & $0.73(0.54-0.99)$ & 0.043 & 0.993 & $0.115^{b}$ & 0.280 & 0.811 & 0.977 & 0.998 \\
\hline rs67756423 C>A & CA vs AA & $0.72(0.55-0.94)$ & 0.017 & 0.996 & $0.045^{b}$ & $0.124^{b}$ & 0.610 & 0.940 & 0.994 \\
\hline \multicolumn{10}{|l|}{ Stratification analysis } \\
\hline \multicolumn{10}{|l|}{$>36$ years } \\
\hline rs12666575T>C & TC vs CC & $0.64(0.41-1.00)$ & 0.048 & 0.861 & $0.148^{b}$ & 0.343 & 0.852 & 0.983 & 0.998 \\
\hline rs1 $107592 A>G$ & AG vs GG & $0.62(0.40-0.96)$ & 0.034 & 0.833 & $0.104^{b}$ & 0.258 & 0.792 & 0.975 & 0.997 \\
\hline rs4976976 A>G & AG vs GG & $0.63(0.40-0.99)$ & 0.045 & 0.842 & $0.138^{b}$ & 0.325 & 0.841 & 0.982 & 0.998 \\
\hline \multicolumn{10}{|l|}{ Male } \\
\hline rs4976976 A>G & $A G$ vs GG & $0.61(0.40-0.93)$ & 0.022 & 0.822 & $0.073^{b}$ & $0.191^{b}$ & 0.722 & 0.963 & 0.996 \\
\hline rs67756423 C>A & CA vs AA & $0.64(0.44-0.92)$ & 0.017 & 0.909 & $0.050^{b}$ & $0.136^{b}$ & 0.635 & 0.946 & 0.994 \\
\hline \multicolumn{10}{|l|}{ Female } \\
\hline rs12666575T>C & TT vs TC + CC & $0.61(0.52-1.15)$ & 0.042 & 0.731 & 0.342 & 0.609 & 0.945 & 0.994 & 0.999 \\
\hline \multirow[t]{2}{*}{ rs1 $107592 A>G$} & AG vs GG & $0.62(0.40-0.95)$ & 0.029 & 0.838 & $0.091^{b}$ & 0.232 & 0.769 & 0.971 & 0.997 \\
\hline & $A A+A G$ vs $G G$ & $0.65(0.43-0.98)$ & 0.038 & 0.895 & $0.118^{b}$ & 0.286 & 0.815 & 0.978 & 0.998 \\
\hline
\end{tabular}

SCZ, schizophrenia; OR: odds ratio; $\mathrm{Cl}$, confidence interval

$p^{\mathrm{a}}<0.05$ indicates statistical significance

${ }^{\mathrm{b}}$ The level of false positive report probability threshold was set at 0.2 and noteworthy findings are presented

This was consistent with our results, which found the TC and TT genotype of rs 12666575 could decrease the risk of SCZ in different subgroups (age $>36$ years old and women, respectively). Rs10275045 is located in the intron region of MAD1L1. A study showed that rs10275045 was associated with SCZ risk in European ancestry [19]. However, the relationship between rs 10275045 and SCZ susceptibility was not observed in the Chinese Han population. One possible reason for the contradiction is the genetic heterogeneity of SCZ in individuals of different ethnic groups. Besides, our results revealed that the AG phenotype of rs1107592 was associated with a lower incidence of SCZ in the overall. Stratification analysis also showed that rs1107592 decreased the susceptibility to SCZ subjects with age $>36$ years. And the AG and AA-AG genotype of rs1107592 played a protective role in SCZ risk of females. To the best of our knowledge, a meta-analysis study has indicated that rs1107592 was related to the susceptibility to $S C Z$, but OR values were not reported [16]. These results demonstrated that MAD1L1 polymorphisms involved in the occurrence of $\mathrm{SCZ}$ and exerted a protective role in SCZ.

TSNARE is located at human chromosome $8 \mathrm{q} 24.3$ and may have a function in intracellular protein transport and synaptic vesicle exocytosis. Recently, the role of TSNARE in SCZ has attracted the attention of researchers. For example, previous analyses showed that TSNARE rs10098073 and rs4129585 were related to
SCZ susceptibility in Caucasians [15]. Similarly, Gu et al. indicated a significant correlation between rs10098073, rs4129585 in TSNARE and SCZ risk in Southeast Chinese Han and Zhuang people [16]. However, there were few studies about TSNARE other polymorphisms and SCZ susceptibility. In the present study, our results discovered that the AG phenotype of rs4976976 and the CA genotype of rs67756423 were related to a lower risk of SCZ in the overall. Then, we further stratified analysis showed that the AG and AA genotype of rs4976976, the CA genotype of rs67756423 decreased the susceptibility to SCZ in males. Additionally, the AG phenotype of rs4976976 reduced the risk of SCZ with age $>36$ years in the heterozygote model. These results indicated that TSNARE polymorphisms are associated with susceptibility to SCZ.

In this study, rs10275045, rs12666575, rs1107592, rs4976976 and rs67756423, located in the intron region of MAD1L1 and TSNARE1, might be associated with the regulation of motifs changed, NHGRI/EBI GWAS hits, GRASP QTL Hits, selected eQTL hits, enhancer histone marks and DNAse, suggesting their potential function in SCZ. In addition, some studies provided evidence to support that intronic SNPs alter the susceptibility to diseases by regulating gene expression [20, 21]. Therefore, we speculated that MAD1L1 and TSNARE polymorphisms may affect the MAD1L1 and TSNARE expression to alter 
the risk of SCZ. However, further study is necessary to confirm this hypothesis.

This work is limited by several factors. First, the sample size of this study was relatively small. A larger sample size was needed to verify our results in future experiments. Second, only three SNPs in MAD1L1 and two SNPs in TSNARE were identified in the present study, and more polymorphisms of these two genes need to be explored. Third, the clinical symptoms of patients, such as severity of disease, were missing. In the future experiments, we should collect complete clinical symptoms of patients to support our findings. Four, although MAD1L1/TSNARE polymorphisms might be associated with SCZ risk, the results were not significant after Bonferroni correction $(p<0.05 / 5)$. Thus, a replication experiment in a cohort with large sample size are required to confirm our findings.

\section{Conclusions}

In conclusion, our findings suggest that genetic polymorphisms in MAD1L1 and TSNARE genes may contribute to risk of SCZ in the Chinese population. These results provide evidence that MAD1L1 and TSNARE may serve as potential biomarkers of SCZ. However, a replication experiment in a cohort with large sample size are required to confirm our findings.

\section{Abbreviations}

SCZ: Schizophrenia; MAD1L1: Mitotic arrest deficient-like 1; TSNARE1: T-SNARE domain-containing 1 gene; OR: Odds ratio; Cl: Confidence intervals; MAF: Minor allele frequency; HWE: Hardy-Weinberg equilibrium; MDR: Multifactor dimensionality reduction.

\section{Supplementary Information}

The online version contains supplementary material available at https://doi. org/10.1186/s12920-021-01070-2.

Additional file 1. Supplemental table 1 Primers used in this study.

\section{Acknowledgements}

We sincerely thank Xi'an Mental Health Center for providing samples for our study.

\section{Authors' contributions}

$\mathrm{XL}$ designed the study protocol and drafted the manuscript; $\mathrm{HX}$ and ZF performed the DNA extraction and genotyping; $\mathrm{QY}$ and TH performed the data analysis; $\mathrm{DZ}, \mathrm{ZL}$, and $\mathrm{HZ}$ performed the sample collection and information recording. All authors read and approved the final manuscript.

\section{Funding}

This study was supported by Hainan Provincial Natural Science Foundation of China (No. 819MS129). The funding bodies had no role in the study design, data collection, analysis and interpretation, or in writing the manuscript.

\section{Availability of data and materials}

The datasets generated and/or analyzed during the current study are available in the figshare repository (https://figshare.com/articles/dataset/Genotype_ $\mathrm{x} \mid \mathrm{s} \times / 14803701)$.

\section{Declarations}

\section{Ethics approval and consent to participate}

The protocol for this study was approved by the Ethics Committee of the Xi'an Mental Health Center and was in line with the Helsinki declaration. The legal guardian of these participants provided informed consent documents on their behalf.

\section{Consent for publication}

Not applicable.

\section{Competing interests}

The authors declare that they have no competing interests.

\section{Author details}

${ }^{1}$ Institute of Mental Health, Hainan Provincial Anning Hospital, No 10, Nanhai Avenue East, Haikou 571100, Hainan, China. ${ }^{2}$ First Department of Psychiatry, Hainan Provincial Anning Hospital, Haikou 571100, Hainan, China. ${ }^{3}$ Department of Nursing, Hainan Provincial Anning Hospital, Haikou 571100, Hainan, China. ${ }^{4}$ Department of Prevention Section, Hainan Provincial Anning Hospital, Haikou 571100, Hainan, China.

Received: 30 March 2021 Accepted: 23 August 2021

Published online: 04 September 2021

\section{References}

1. Kempisty B, Bober A, Łuczak M, Czerski P, Szczepankiewicz A, Hauser J, et al. Distribution of 1298A >C polymorphism of methylenetetrahydrofolate reductase gene in patients with bipolar disorder and schizophrenia. Eur Psychiatry. 2007:22:39-43.

2. Saha S, Chant D, Welham J, McGrath J. A systematic review of the prevalence of schizophrenia. PLoS Med. 2005;2:e141.

3. Chan KY, Zhao F-F, Meng S, Demaio AR, Reed C, Theodoratou E, et al. Prevalence of schizophrenia in China between 1990 and 2010. J Global Health. 2015;5:010410.

4. Lichtenstein P, Yip BH, Björk C, Pawitan Y, Cannon TD, Sullivan PF, et al. Common genetic determinants of schizophrenia and bipolar disorder in Swedish families: a population-based study. Lancet. 2009;373:234-9.

5. Sullivan PF, Kendler KS, Neale MC. Schizophrenia as a complex trait: evidence from a meta-analysis of twin studies. Arch Gen Psychiatry. 2003;60:1187-92

6. Cardno AG, Gottesman II. Twin studies of schizophrenia: from bow-andarrow concordances to star wars Mx and functional genomics. Am J Med Genet. 2000;97:12-7.

7. Chen RH, Brady DM, Smith D, Murray AW, Hardwick KG. The spindle checkpoint of budding yeast depends on a tight complex between the Mad1 and Mad2 proteins. Mol Biol Cell. 1999;10:2607-18.

8. Sun Q, Zhang X, Liu T, Liu X, Geng J, He X, et al. Increased expression of mitotic arrest deficient-like 1 (MAD1L1) is associated with poor prognosis and insensitive to Taxol treatment in breast cancer. Breast Cancer Res Treat. 2013;140:323-30.

9. Li D, Meng Q, Zhang H, Feng T, Liu M, Cai L. Mitotic arrest deficient-like 1 is correlated with poor prognosis in small-cell lung cancer after surgical resection. Tumour Biol. 2016;37:4393-8.

10. Trost S, Diekhof EK, Mohr H, Vieker H, Krämer B, Wolf C, et al. Investigating the impact of a genome-wide supported bipolar risk variant of MAD1L1 on the human reward system. Neuropsychopharmacology. 2016:41:2679-87.

11. Whelan R, St Clair D, Mustard CJ, Hallford P, Wei J. Study of novel autoantibodies in schizophrenia. Schizophr Bull. 2018;44:1341-9.

12. Zhao L, Chang H, Zhou D-S, Cai J, Fan W, Tang W, et al. Replicated associations of FADS1, MAD1L1, and a rare variant at 10q26.13 with bipolar disorder in Chinese population. Transl Psychiatry. 2018;8:270. 
13. Smith JJ, Sumiyama K, Amemiya CT. A living fossil in the genome of a living fossil: harbinger transposons in the coelacanth genome. Mol Biol Evol. 2012;29:985-93.

14. Sinzelle L, Kapitonov WV, Grzela DP, Jursch T, Jurka J, Izsvák Z, et al. Transposition of a reconstructed Harbinger element in human cells and functional homology with two transposon-derived cellular genes. Proc Natl Acad Sci USA. 2008;105:4715-20.

15. Sleiman P, Wang D, Glessner J, Hadley D, Gur RE, Cohen N, et al. GWAS meta analysis identifies TSNARE1 as a novel Schizophrenia/Bipolar susceptibility locus. Sci Rep. 2013;3:3075.

16. Gu LZ, Jiang T, Cheng ZH, Zhang YC, Ou MM, Chen MC, et al. TSNARE1 polymorphisms are associated with schizophrenia susceptibility in Han Chinese. J Neural Transm (Vienna). 2015;122:929-32.

17. Wacholder S, Chanock S, Garcia-Closas M, El Ghormli L, Rothman N. Assessing the probability that a positive report is false: an approach for molecular epidemiology studies. J Natl Cancer Inst. 2004;96:434-42.

18. Bergen SE, O'Dushlaine CT, Ripke S, Lee PH, Ruderfer DM, Akterin S, et al. Genome-wide association study in a Swedish population yields support for greater CNV and MHC involvement in schizophrenia compared with bipolar disorder. Mol Psychiatry. 2012;17:880-6.

19. Ruderfer DM, Fanous AH, Ripke S, McQuillin A, Amdur RL, Schizophrenia Working Group of the Psychiatric Genomics C, et al. Polygenic dissection of diagnosis and clinical dimensions of bipolar disorder and schizophrenia. Mol Psychiatry. 2014;19:1017-24.

20. Zhao H, Yang W, Qiu R, Li J, Xin Q, Wang X, et al. An intronic variant associated with systemic lupus erythematosus changes the binding affinity of Yinyang1 to downregulate WDFY4. Genes Immun. 2012;13:536-42.

21. Wang D, Sadee W. CYP3A4 intronic SNP rs35599367 (CYP3A4*22) alters RNA splicing. Pharmacogenet Genom. 2016;26:40-3.

\section{Publisher's Note}

Springer Nature remains neutral with regard to jurisdictional claims in published maps and institutional affiliations.
Ready to submit your research? Choose BMC and benefit from:

- fast, convenient online submission

- thorough peer review by experienced researchers in your field

- rapid publication on acceptance

- support for research data, including large and complex data types

- gold Open Access which fosters wider collaboration and increased citations

- maximum visibility for your research: over $100 \mathrm{M}$ website views per year

At BMC, research is always in progress.

Learn more biomedcentral.com/submissions 